\title{
Application of image processing to Computer Graphics*
}

\author{
Chen Chunyu ${ }^{1, a}$, Wang Fucheng ${ }^{2, b}$, Chen Xin $^{1}$, Cui Feng ${ }^{1}$, Zhang Lili ${ }^{1}$, \\ Zhang Chen ${ }^{3}$ \\ ${ }^{1}$ Department of Physical and Electricity Information Engineering, Daqing Normal University, Daqing, \\ Heilongjiang, China. \\ ${ }^{2}$ College of Engineering, Heilongiiang Bayi Agricultural University, Daqing, Heilongjiang, China. \\ ${ }^{3}$ College of Electronic Science, Northeast Petroleum University, Daqing, Heilongjiang,China. \\ aemail: ccy08190309@163.com, bemail: fuchengwang@163.com
}

Keywords: Image processing; Computer Graphics; Image extracting; Automatic marking

\begin{abstract}
The examination of the Computer Graphics is basically the computer examination to investigate the drawing ability in universities in recent years. Based on many years of teaching practice and according to the transformation trend of the computer intelligent paper marking, the image processing technology is adopted, and the key information of the image is extracted, and the image similarity calculation program is compiled, and the CAD automatic paper marking function is implemented by the contrast of the students' plots with the standard answer. Through the calculation examples, the grading results are consistent with the artificial results ideally. The calculation speed is faster than the manual speed, thus the teacher's working efficiency is improved.
\end{abstract}

\section{Introduction}

Most examinations of the Computer Graphics are the computer examinations. The traditional correcting paper way is to print the students' electronic editions out. As a result, the speed is slow, and the efficiency is low, and lots of subjective errors happen. With the development of image processing technology, the technology is applied in the Computer Graphics examinations to implement the automatic grading, so the working efficiency is improved. At present, the automatic marking way has become a trend in the university's plot test, and it adapts to the needs of the major construction and the curriculum development ${ }^{[1-4]}$.

\section{The Application of Image Processing to Automatic Marking}

To get the strong operational capacity of MATLAB in the matrix calculation, we use MATLAB to process students' plots, which are expressed by matrix and vector.

During the actual exam, students mainly copy the fixed drawing. For the completed CAD plots, we can make them become the graphics format by using the CAD print function. Thus we can calculate the similarity degree to get students' scores. The similarity degree calculation mainly depends on the similarity between two images' content, so it determines the score. To measure the image similarity is based on the difference between the mathematical vectors to measure the image similarity. At present, the method is adopted more than others because using histogram can realize the normalization better, and the computation is less, and the speed is faster ${ }^{[4-6]}$.

The formula is

$G$ is the target image histogram. $S$ is the source image histogram. $N$ is the number of samples

$$
\operatorname{Sim}(G, S)=\frac{1}{N} \sum_{i=1}^{N}\left(1-\frac{\left|g_{i}-s_{i}\right|}{\operatorname{Max}\left(g_{i}, s_{i}\right)}\right)
$$

\footnotetext{
*1.Youth Foundation of Daqing Normal University research project (Serial number: 09ZQ08)

2.Higher Education Reform project in Heilongjiang Province. Standy on the Teaching Mode and Practice of Mechanical drawing. (Serial number: JG2012010455)
} 
of the color space. $g_{i}$ is the image property of the target image sub- block area. $s_{i}$ is the image property of the source image sub- block area.

The histograms only describe the distribution of colors in the image global, but it can't describe the location distribution and the positions of the colors ${ }^{[7-10]}$. So the image is divided into sub-blocks regularly, and the corresponding similarity degrees of the small pieces are calculated. As result, the average similarity degree of the small reflects the whole similarity degree. After such modification, the algorithm has been capable of reflecting the local distribution and the position of the color in a certain extent. Thus it can compensate for the lack of the global histogram algorithm better.

\section{The Processing of Cad By Matlab}

For CAD Exam on computer, most teachers adopt a subjective papermarking way. The scoring criterion of the way is inconsistent. Papers are marked according tothe same criteria, and the fair score management system is established. Using the image processing of MATLAB compares the students' plots with the standard answers to get students' scores.

The first step of MATLAB image processing is to print the students' plots into picture formats. Because a lots of students' drawings lead to large Manual print workload, using the batch printing program edited by VB a built-in tools completes batch printing. The batch printing of CAD is shown in Fig.1.

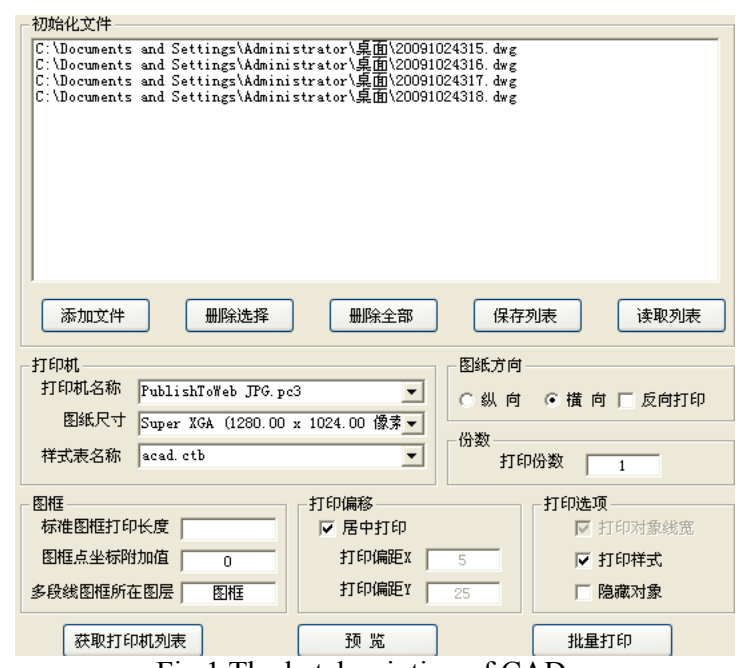

Fig.1 The batch printing of CAD

The second step is to use the program edited by MATLAB to calculate the similarity degree between the picture processed and the standard drawing. Thus the scores can be gotten. The program flowchart is Fig.2.

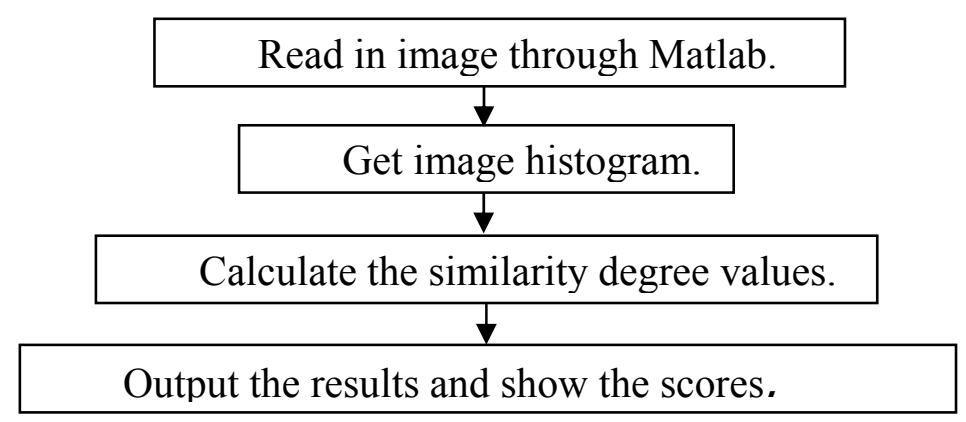

Fig.2 The program flowchart

\section{Application Examples}

In the actual process of computer graphics exam, three more different plots drawn are chosen to compare with the standard drawing. In order to verify the legitimacy of the program, we use the batch printing program of CAD to print the three drawings into picture formats, and print them into 
the same norm of the standard drawing, and store them in a single file folder, and get the corresponding scores by the similarity degree calculation program. The program reads in the three pictures and calculates the results. The results are shown in Fig.3, in Fig.4, in Fig.5.
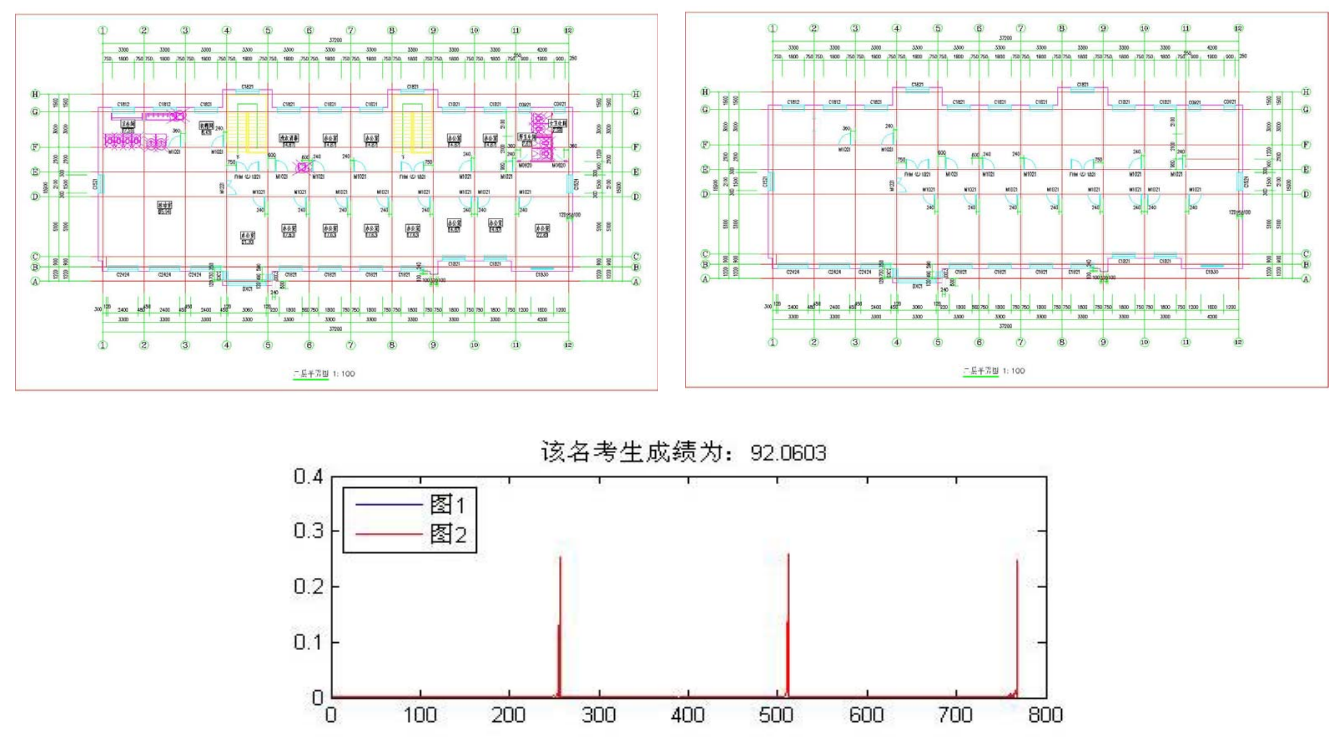

Fig.3 The drawing completed well
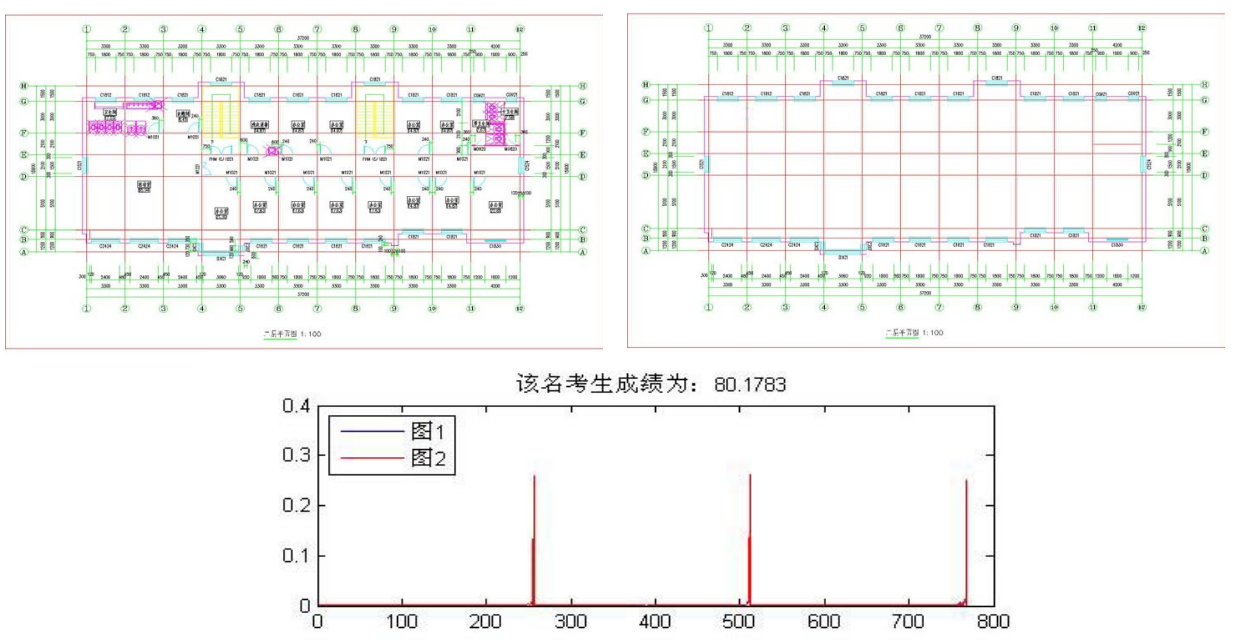

Fig.4 The drawing completed basically
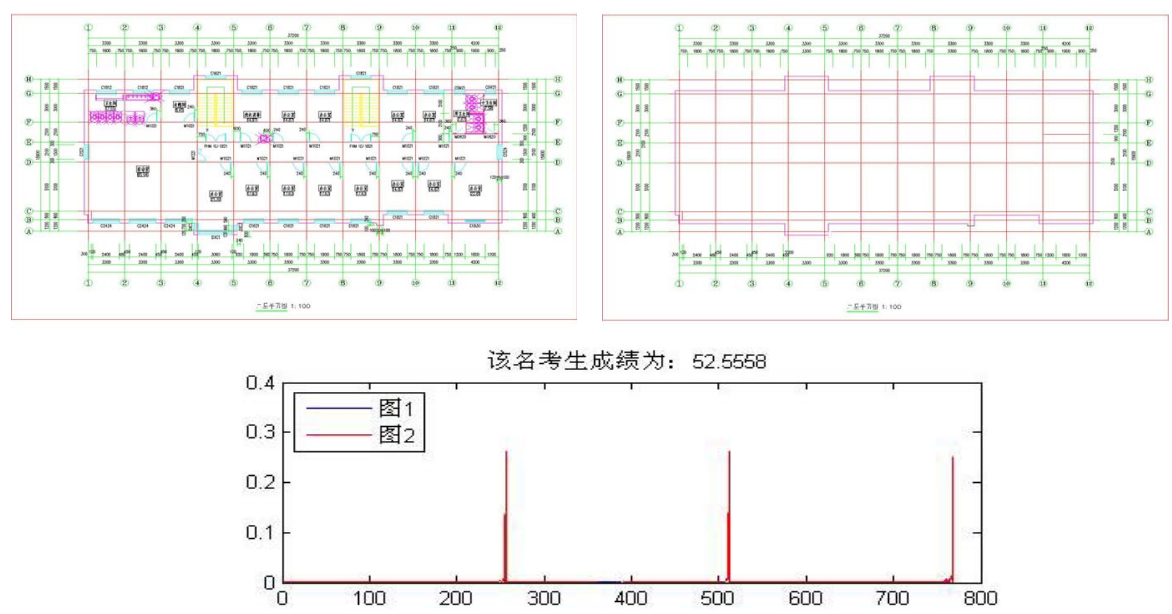

Fig.5 The drawing completed partly

Comparing the three output results with manual marking, they are consistent by point rounding basically. Selecting different drawings and repeating tests, we can get the same conclusion. 


\section{Conclusion}

Based on image similarity calculation principles, using the image process of MATLAB, editing the graphics similarity calculation program, comparing the CAD drawings by batch printing with the standard answer, we can draw the conclusion that the method can get a ideal scores closing to the manual score and accomplish the CAD automatic paper marking function. The speed of the method is faster than before, and it can save more time. Thus it has a good prospect for practical application.

\section{References}

[1]Wang Like. Application of image processing and recognition based on online marking [D]. Shandong: Shandong university, 2005.

[2]Song Zhengzheng. Research and realization of automatic scan paper marking system [D]. Beijing: North china electric power university（Beijing）,2008.

[3]Zhao Xiaojuan. Research on recognition method of handwritten numbers and English characters[D]. Changchun: Northeast normal university,2010.

[4]Liu Xin, Yang Hongpei. The research on organization of digital image and retrieval based on gray histogram and project[J]. Journal of xuchang university, 2007, 26(2): 86-90.

[5]Gao Yupeng, Yang Jun, He Guangjun. Research on auto-grading system based on Image Identification Technology [J]. Modern electronics Technique. 2006, 22(237): 119-120.

[6]Xu Lina, Peng Yuanhua. The modified graph cut model for image segment[J]. Science technology and engineering.2006,6(18):2854-2857.

[7]Yasuhiro Ajiro, Kazunori Ueda. Kima: An automated error correction system for concurrent logic programs[J]. Automated software engineering,2002,(9):68-94.

[8]Zhang Song. Research of image mosaic technology [D]. Harbin: Harbin institute of technology,2008.

[9]Xiao Lifeng, Yang Yongyue. Image processing system in the role of online marking[J]. Computer knowledge and technology ,2009,5(3):703-705.

[10]Ruan Shaolin. Research of information extraction and recognition technology in online marking system[D]. University of electronic science and technology of china, 2010. 